\title{
The ion experiment onboard the Interball-Aurora satellite; initial results on velocity-dispersed structures in the cleft and inside the auroral oval
}

\author{
J. A. Sauvaud ${ }^{1}$, H. Barthe ${ }^{1}$, C. Aoustin ${ }^{1}$, J. J. Thocaven ${ }^{1}$, J. Rouzaud ${ }^{1}$, E. Penou ${ }^{1}$, D. Popescu ${ }^{1}$, R. A. Kovrazhkin ${ }^{2}$, \\ K. G. Afanasiev ${ }^{2}$ \\ ${ }^{1}$ Centre d'Etude Spatiale des Rayonnements, BP-4346, F-31029, Toulouse, France \\ e-mail:sauvaud@cesr.cnes.fr \\ ${ }^{2}$ Space Research Institute, Profsoyuznaya 84/32, Moscow, Russia
}

Received: 10 October 1997 / Revised: 27 February 1998 / Accepted: 10 March 1998

\begin{abstract}
The Toulouse ION experiment flown on the Russian Interball-Aurora mission performs simultaneous ion and electron measurements. Two mass spectrometers looking in opposing directions perpendicular to the satellite spin axis, which points toward the sun, measure ions in the mass and energy ranges 1-32 amu and $\sim 0-14000 \mathrm{eV}$. Two electron spectrometers also looking in opposing directions perform measurements in the energy range $\sim 10 \mathrm{eV}-20000 \mathrm{eV}$. The InterballAurora spacecraft was launched on 29 August 1996 into a $62.8^{\circ}$ inclination orbit with an apogee of $\sim 3 \mathrm{R}_{\mathrm{E}}$. The satellite orbital period is $6 \mathrm{~h}$, so that every four orbits the satellite sweeps about the same region of the auroral zone; the orbit plane drifts around the pole in $\sim 9$ months. We present a description of the ION experiment and discuss initial measurements performed in the cusp near noon, in the polar cleft at dusk, and inside the proton aurora at dawn. Ion-dispersed energy structures resulting from time-of-flight effects are observed both in the polar cleft at $\sim 16$ hours MLT and in the dawnside proton aurora close to 06 hours MLT. Magnetosheath plasma injections in the polar cleft, which appear as overlapping energy bands in particle energy-time spectrograms, are traced backwards in time using a particle trajectory model using 3D electric and magnetic field models. We found that the cleft ion source is located at distances of the order of $18 \mathrm{R}_{\mathrm{E}}$ from the earth at about 19 MLT, i.e., on the flank of the magnetopause. These observations are in agreement with flux transfer events (FTE) occurring not only on the front part of the magnetopause but also in a region extending at least to dusk. We also show that, during quiet magnetic conditions, time-of-flight ion dispersions can also be measured inside the dawn proton aurora. A method similar to that used for the cleft is applied to these auroral energy dispersion signatures. Unexpectedly, the ion source is found to be at distances of the order
\end{abstract}

Correspondence to: J. A. Sauvaud of $60-80 R_{E}$, at the dawn flank of the magnetosphere. These results are discussed in terms of possible entry, acceleration, and precipitation mechanisms.

Key words. Magnetospheric physics · Auroral phenomena - Energetic particles - Magnetopause, cusp, and boundary layers · Interball-Aurora satellite.

\section{Introduction}

Direct measurements of suprathermal plasma in the earth's magnetosphere are essential for the understanding of the filling of the magnetosphere with ionospheric and solar wind plasmas, for the processes at the root of electromagnetic energy dissipation in the auroral zones, and for solar wind energy accumulation in the magnetospheric tail. Simultaneous measurements performed in key regions such as the ionosphere and the magnetospheric outer boundaries are critical for understanding the dynamics of the complete system. Such simultaneous large-scale measurements are one of the main aims of the Interball project, which consists of two widely spaced main satellites, the tail probe (TP) (apogee $\sim 200000 \mathrm{~km}$ ) and the auroral probe (AP) (apogee $\sim 20000 \mathrm{~km}$ ), the tail probe being associated with a subsatellite for mesoscale measurements (Galeev et al., 1996).

The ION instrument flown onboard the AP satellite was developed to measure auroral ions and electrons. This instrument allows detailed studies of :

- the dynamics of the polar cap and of the auroral zone plasma and field under the influence of interplanetary medium changes and internal processes such as substorms;

- the acceleration and precipitation mechanisms of electrons and ions $\left(\mathrm{H}^{+}, \mathrm{He}^{+}, \mathrm{He}^{++}, \mathrm{O}^{+}\right)$in discrete 
and diffuse structures and especially at the footprint of the magnetospheric boundaries;

- the local heating of ions from the ionosphere and their ejection into the magnetosphere;

- the ionospheric signatures of the acceleration and transport of suprathermal particles at the magnetopause and in the magnetospheric tail;

- the processes of turbulent heating and acceleration of particles by powerful ground-based VLF-transmitters.

In this paper, after a description of the experiment, we focus on the ionospheric signatures of the solar plasma entry and acceleration processes in the magnetosphere using mainly hydrogen and electron data. Other initial ION data pertaining in particular to ionospheric ion outflow can be found in the companion paper by Dubouloz et al. (1998).

\section{Instrument description}

\subsection{Basic measurement capabilities}

The ION instrument consists of two energetic ion composition spectrometers (ION-1 and ION-2), and of two energetic electron spectrometers (ION-3 and ION4). Figure 1 shows the block diagrams of the sensor heads. The two ion and two electron spectrometers view opposite directions, perpendicular to the spin axis of the satellite, which is directed toward the sun. However the viewing directions of the ion and electron spectrometers are different. The spin period of the satellite is $\sim 120 \mathrm{~s}$.

ION-1 and ION-2 provide routine measurements of $\mathrm{H}^{+}$and $\mathrm{O}^{+}$, scanned successively either in the energy range $\sim 0-14000 \mathrm{eV} / Q$ or in the range $\sim 0-120 \mathrm{eV} / Q$, where $Q$ is the ion charge. Four ions, $\mathrm{H}^{+}, \mathrm{He}^{++}, \mathrm{He}^{+}$, and $\mathrm{O}^{+}$, or all masses between 1 and $64 \mathrm{amu}$, can also be measured. The geometrical factors of the two spectrometers are identical and depend on the energy, ranging from 2 to $6 \times 10^{-3} \cdot E(\mathrm{keV}) \mathrm{cm}^{2} \cdot \mathrm{ster} \cdot \mathrm{keV}$ where $E$ is the ion energy. The mass resolution at the focus, $M / \Delta M$, is greater than 10 (Fig. 2).

ION-3 and ION-4 perform measurements of differential electron fluxes in the energy range 10-20 $000 \mathrm{eV}$. Both electron spectrometers are identical. Each one uses two different electron multipliers (channeltrons) at the output of the electrostatic analyzer, each channeltron providing a different geometrical factor: $1 \times 10^{-5} \cdot E(\mathrm{keV})$ and $8 \times 10^{-5} \cdot \mathrm{E}(\mathrm{keV})\left(\mathrm{cm}^{2} \cdot \mathrm{ster} \cdot \mathrm{keV}\right)$.

The experiment electronics includes a low-voltage power converter, and a data processing unit, based on an 80C86 CPU in charge of the data acquisition and compression and the interface with the telemetry systems. The DPU also commands the electronics for the sensor operational modes and the devices for communication with the onboard magnetometer and with the satellite service systems.

\subsection{Ion composition analyzers}

The design of the detector heads of the ION-1 and ION2 mass spectrometers is based on the principle of the
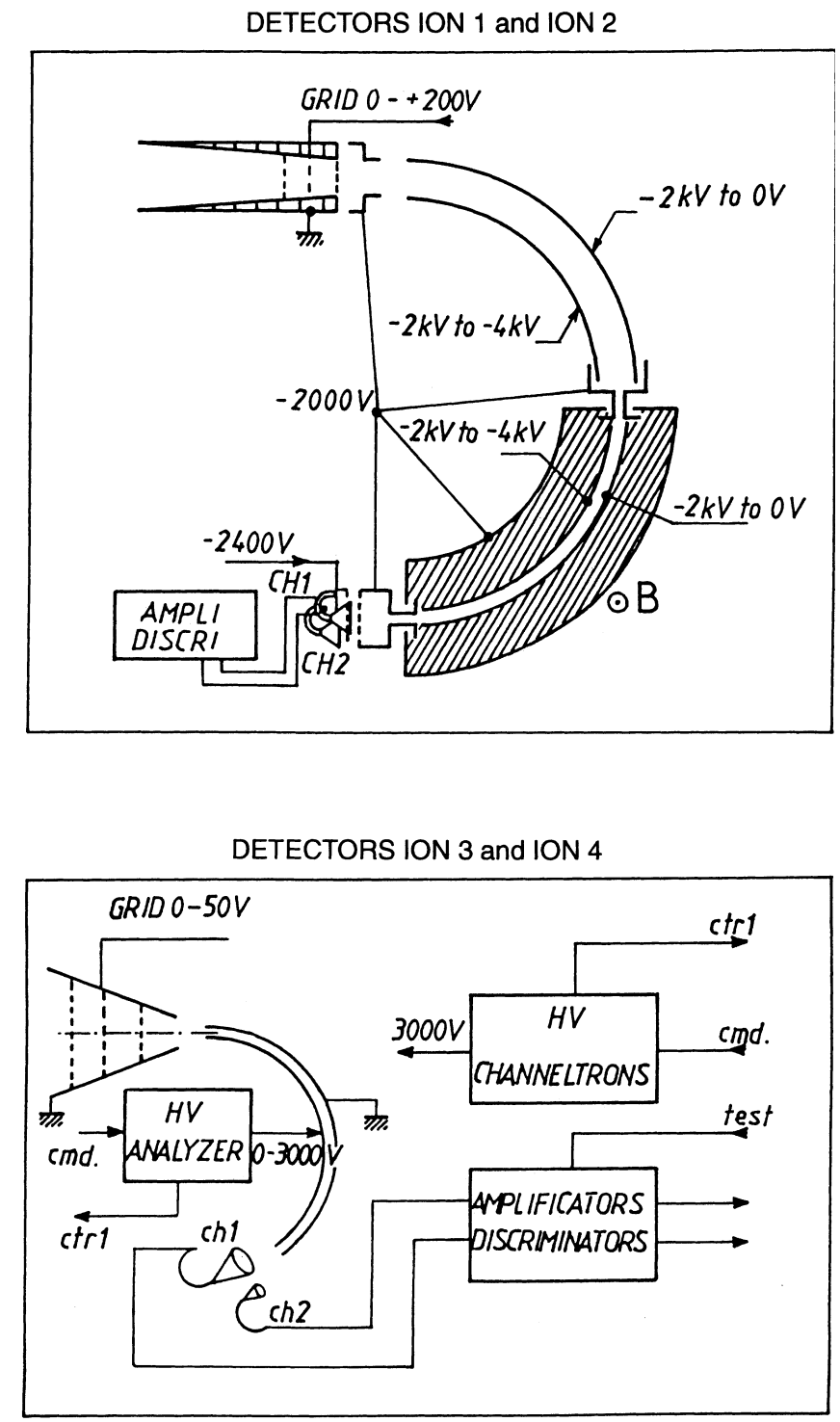

Fig. 1. Block diagram of the ion mass spectrometers (top) and the electron sensors (bottom)

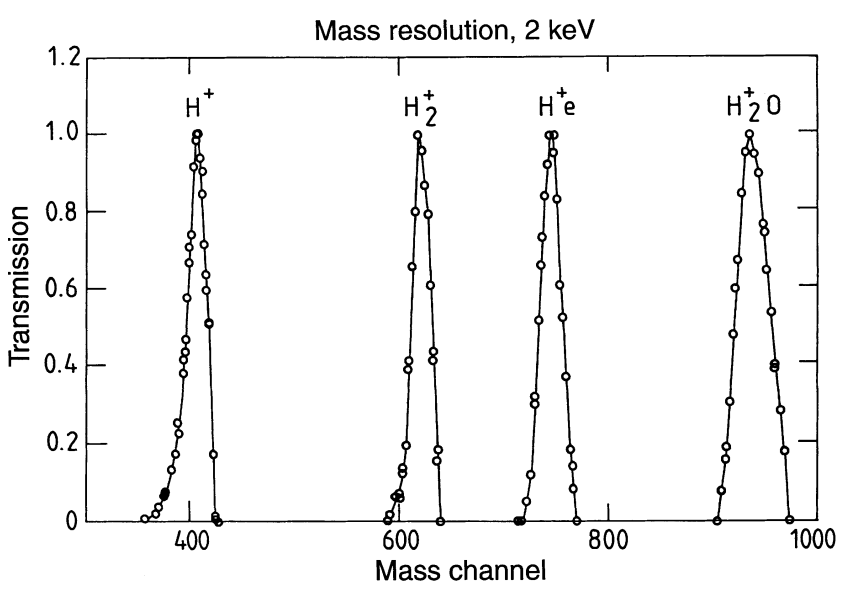

Fig. 2. Mass resolution of the $\mathrm{ION}-1 / 2$ spectrometers for $2-\mathrm{keV}$ particles (focus) 
Wien filter, where the mass filtering is performed by crossed electric and magnetic fields (e.g., Ghielmetti, 1974). The energy over charge $(E / Q)$ selection is made by a cylindrical electrostatic analyzer placed in front of the filter (Fig. 1). The design of the ION-1 and ION-2 sensors is quite similar to that of the mass spectrometers previously flown onboard GEOS-1/2, ISEE-1, DE-1, AUREOL-3, and AMPTE/CCE (Balsiger et al., 1976; Shelley et al., 1978, 1981, 1985; Bosqued et al., 1982). Particles enter the ion spectrometers through a collimator. All positive ions are then accelerated through a potential of $-2000 \mathrm{~V}$. The remainder of the analysis system is maintained at this negative centerline potential. Therefore, ions enter the first electrostatic analyzer with an energy per charge $E_{\text {int }}(\mathrm{eV})=E_{\text {ext }}+2000$. This analyzer selects particles with a ratio $E_{\text {int }} / V=4.94$. The voltage applied to the analyzer plates is programmable in 32 steps allowing the subdivision of the energy range from 2000 to $16000 \mathrm{eV} / Q$ internal to the analyzer. In terms of ion energy outside the analyzer, the mean particle energy per charge is given by:

$$
E_{\infty}=\left[2030(1.068866)^{i}-2000\right] \mathrm{eV} / \mathrm{e}, \quad 0 \leq i \leq 31 .
$$

The FWHM energy pass-band is $\sim 4 \%$ of the internal energy (Fig. 3) so that:

$$
\Delta E_{\infty}(F W H M)=80 \times(1.068866)^{i} \mathrm{eV} / Q, \quad 0 \leq i \leq 3 .
$$

$\Delta E_{\infty} / E_{\infty}$ increases greatly at low energies and for $i=0$, all ions with energies between the satellite potential and $\sim 70 \mathrm{eV}$ are selected by the electrostatic analyzer. Between the end of the collimator and the entrance of the electrostatic analyzer, a retarding potential analyzer

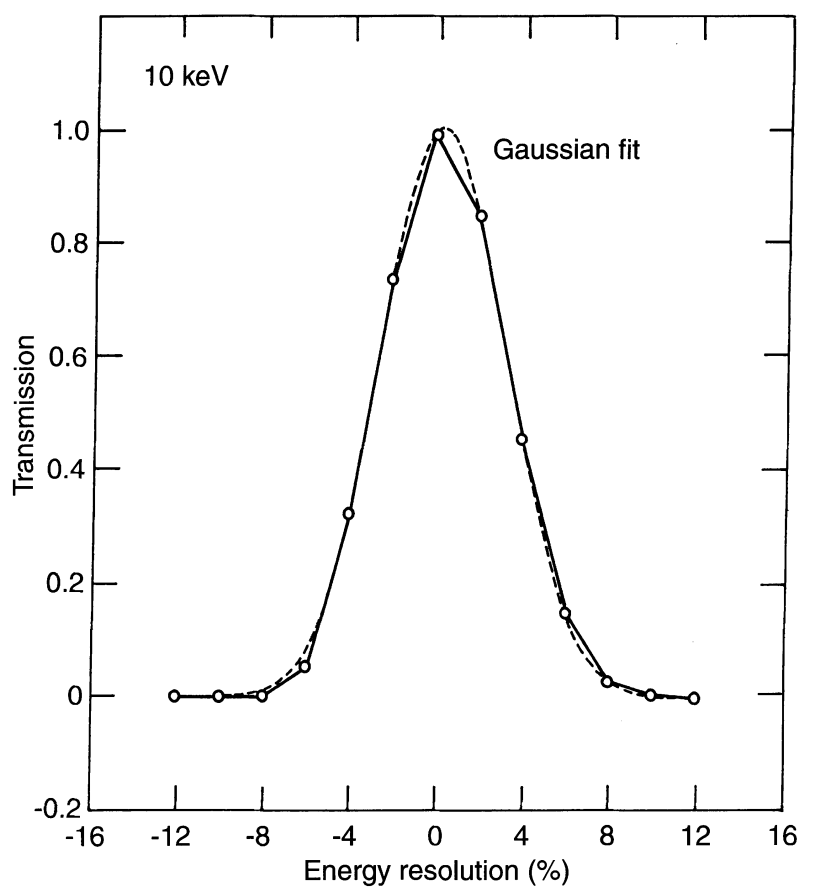

Fig. 3. Energy resolution of the ION-2 mass spectrometer at high energy $(10 \mathrm{keV})$
(RPA) can be switched on to subdivide this energy range. The RPA can be used to select ions into 31 energy bands between $V_{\mathrm{RPA}, i}$ and $70 \mathrm{eV}$ ( $V_{\mathrm{RPA}, i}$ being the voltage applied to the RPA central grid). However this mode is rarely used, the Hyperboloid experiment being dedicated to the analysis of ions with energies $<80 \mathrm{eV}$ (Dubouloz et al., 1998). After $E / Q$ selection ions undergo an $M / Q$ selection in the magnetic analyzer, which consists of a second cylindrical electrostatic deflection system placed between the poles of a Samarium-Cobalt magnet, with a Fe-Co yoke, giving a $\sim 1000$ $\mathrm{G}$ field. The $E$ and $B$ fields are nearly perpendicular and arranged so that their deflections on the ions are additive. The equation of motion for a normally incident ion, traveling along the central line of the magnetic analyzer, is:

$2 E_{\text {int }} / R=\xi(R)+\left(2 Q E_{\text {int }} / M\right)^{1 / 2} B$,

where $\xi$ is the radial electric field at $R, Q$ is the ion charge, $M$ the ion mass, and $B$ the magnetic field. The internal energy per charge $E_{\text {int }}$ is fixed by the first electrostatic analyzer. At each energy step, the electric field in the magnetic analyzer is programmed in order either to scan 2 or 4 ions $\left(\mathrm{H}^{+}, \mathrm{He}^{++}, \mathrm{He}^{+}, \mathrm{O}^{+}\right)$or to step through 64 values in such a way that the same mass always appears at the same step number, independently of the ion energy per charge. At the end of the selection system the ion strikes one of the two channeltrons used to multiply the particle charge. Amplifiers convert the charge, collected at the outputs of the channeltrons, into a detectable digital pulse. The count rate delivered by one or two channeltrons during each mass step is sent to the telemetry system. The mass spectrometers have been calibrated in the CESR vacuum facilities using a large ion beam to obtain the energy, angle, and mass transmissions at various energies, and to construct the voltage tables necessary for the flight software. Figures 2 and 3 illustrate the main characteristics of the spectrometers, i.e., the mass separation ability at the focus and the energy resolution at high energy from which, using Eq. (3), the energy resolution at low energies can be deduced.

\subsection{Electron analyzers}

Two spectrometers, ION-3 and -4 , measure electron fluxes in the energy range $\sim 10-20000 \mathrm{eV}$. They consist of two $127^{\circ}$ cylindrical electrostatic analyzer heads mounted on the spinning spacecraft body with (1) fields of view instantaneously opposed (anti-collinear) to each other, (2) narrow, symmetrical energy bandwidth of $\Delta E / E=6 \%$, (3) narrow field of view, and (4) two directional geometrical factors. These different values result from the use of two different channeltrons at the output of each analyzer. Each channeltron has its associated amplifier and discriminator (Fig. 1).

The outer analyzer plate of the analyzers is grounded, while the inner one sweeps positive potentials, from $\sim 1$ to $\sim 2700 \mathrm{~V}$. The analyzer constant is $k=E / \mathrm{V}=7.5(\mathrm{eV} / \mathrm{V})$. The electron energy range is 

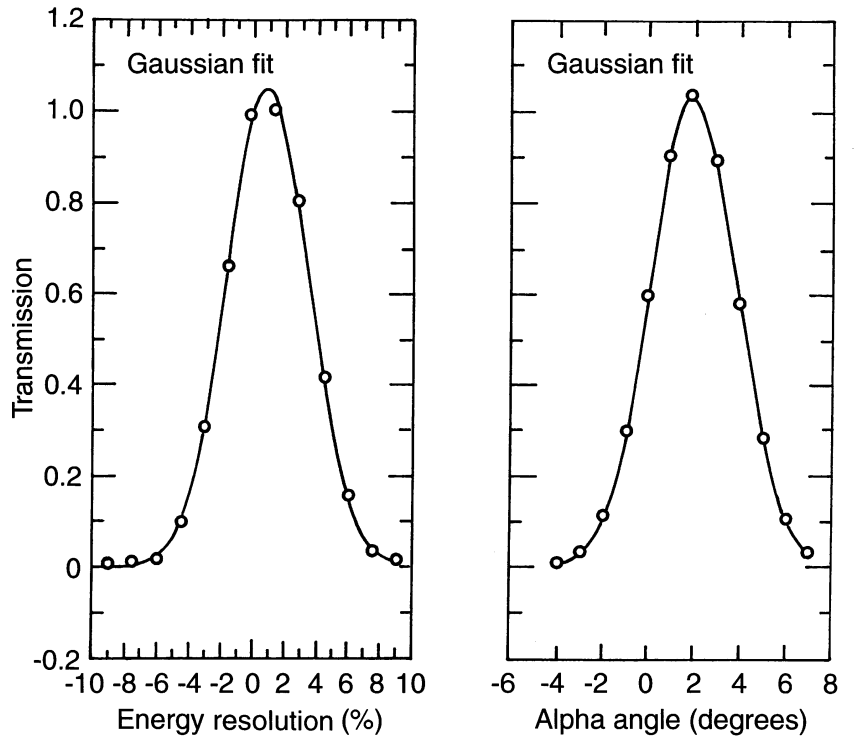

scanned in 128 elementary steps. The count rate, for 32 energy steps summed over four consecutive elementary steps, is sent to the telemetry systems. Charge amplifiers are used to convert the charge, collected at the outputs of each channeltron, into a detectable voltage pulse. The spectrometers have been calibrated to obtain the transmission as a function of energy and angle. Figure 4 gives the main results of the calibration, i.e., the energy and angular resolutions.

\subsection{Operating modes}

The mass spectrometers ION-1 and -2 can be operated in 31 different modes:

25 energy modes to measure the energy range from $\sim 30$ to $14000 \mathrm{eV}$. During each energy step 1, 2, or 4 different ions are analyzed. The mass analysis voltage can be either a single value or made up of three closely spaced steps.

1 energy mode to step 64 values of the mass range between 1 and 64 amu.

4 modes during which the voltage of the central grid of the RPA varies to allow the detection of cold ions.

2 test modes to allow the verification of the experiment.

Each mode can be spin-synchronized or free running. The count rates delivered by each of the two channeltrons of each spectrometer can be transmitted to the telemetry. It is also possible to transmit the information coming from a single channeltron or the sum of the two channeltrons. Energy spectra have 8, 16, or 32 channels, and 16,32 , or 64 spectra per spin are sampled. Furthermore, during each spin, 16 magnetic field vectors are transmitted to the ION sensor from the onboard magnetometer. Moreover, during direct telemetry sessions, the ION experiment sends 8,4 , or 2 magnetic field vectors per second to the telemetry.

The electron detectors can be operated in three main modes, with 128,64 , or 32 energy sweeps per spin. The

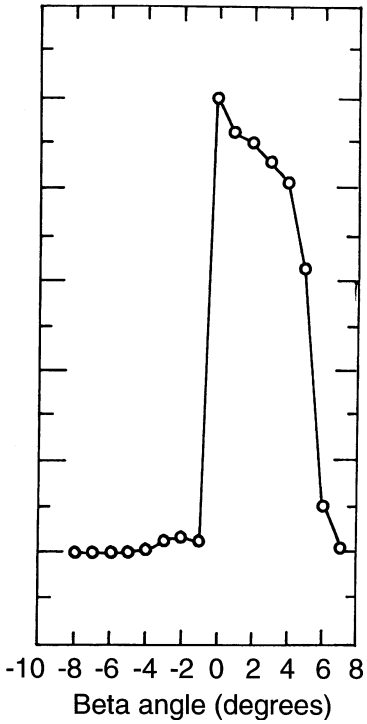

Fig. 4. Energy and angular resolutions of the electron detectors ION-3/ 4. The angle $\alpha$ is measured in a plane perpendicular to the analyzer cylindrical plates; $\beta$ is measured in a plane perpendicular to this

energy table can be changed by command to avoid the detection of high fluxes of photoelectrons. Furthermore at the entrance of the analyzer, a set of grids can be used to repel secondary electrons.

\section{Scientific results}

As the first scientific results, we will present (1) examples of cusp crossings near noon, (2) cleft measurements showing time-dependent magnetosheath plasma injections near 16 hours MLT, and (3) $\mathrm{keV}$ quiet-time auroral proton precipitation characterized by time-offlight signatures. Superposed ion dispersion curves in the cleft are shown to be indicative of pulsed magnetic reconnection acting on the side of the magnetosphere (MLT $=19$ hours, $R=18 \mathrm{R}_{\mathrm{E}}$ ). The ion energy dispersions inside the proton aurora are similarly used to estimate the source distance in the tail which, unexpectedly, is located very far from the earth, at about $80 \mathrm{R}_{\mathrm{E}}$ on the dawn flank of the tail. These results are discussed in terms of possible entry, acceleration, and precipitation mechanisms.

\subsection{Dayside polar cusp}

A typical polar cusp crossing during a southward IMF episode $\left(B_{z} \sim-5 \mathrm{nT}\right)$ is presented in Fig. 5. Data are taken along orbit 791 on 7 March 1997 from 18:00 to 18:30 UT, when Interball-Aurora flew from the dayside auroral regions to the nightside. From the top to the bottom, Fig. 5 presents the energy-time spectrogram of electrons in the energy range from 30 to $20000 \mathrm{eV}$, the energy-time spectrogram of hydrogen ions in the energy range from 30 to $14000 \mathrm{eV}$, and their pitch angle. Fluxes expressed in particles $\left(\mathrm{cm}^{2} \cdot \mathrm{s} \cdot \mathrm{ster} \cdot \mathrm{keV}\right)^{-1}$ are color coded. Several characteristic features are apparent: 

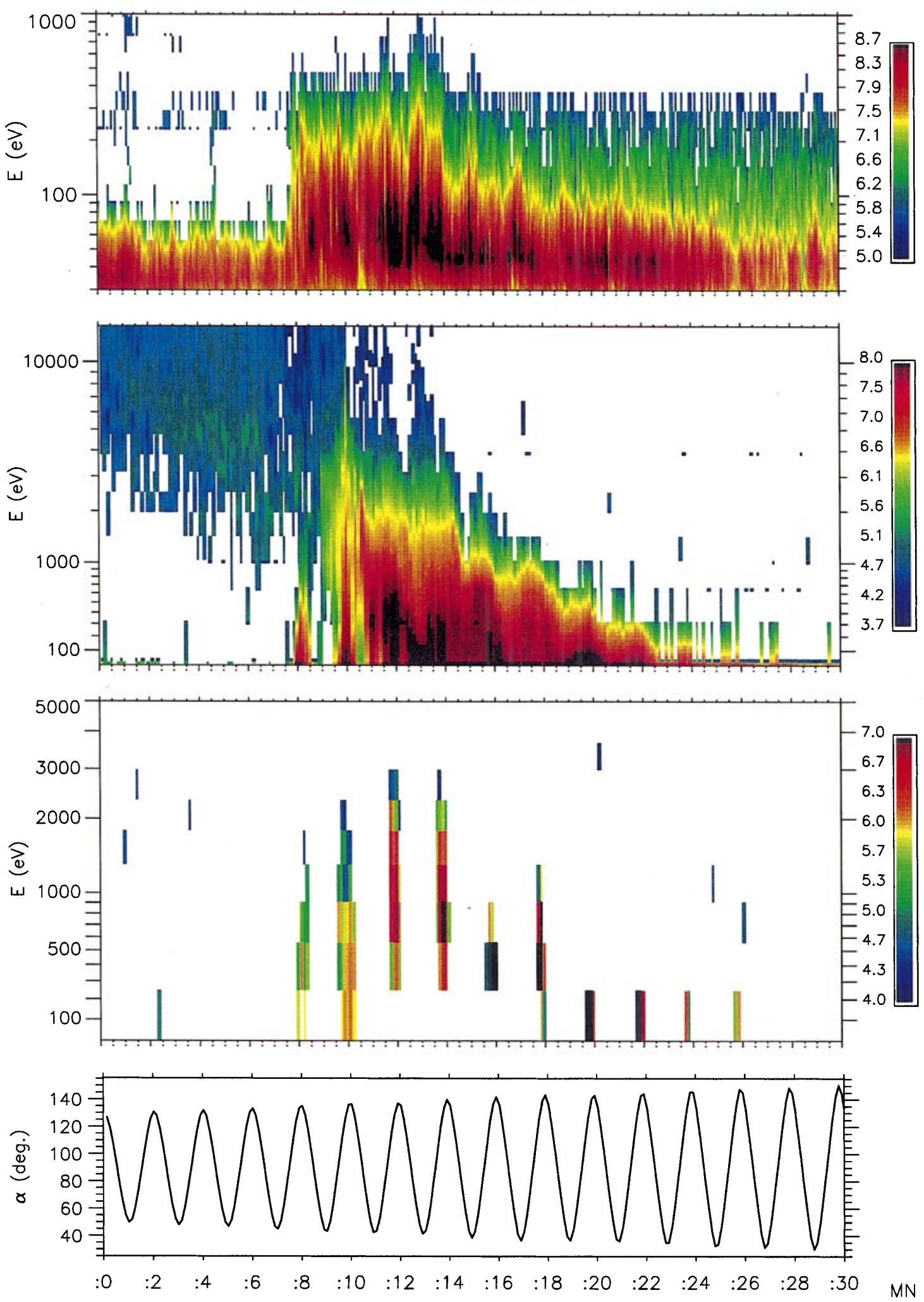

$\begin{array}{rr}H & 1.74 \\ \Lambda_{0} & 73.07 \\ M L T & 11.98 \\ \text { LONG } & 276.86\end{array}$
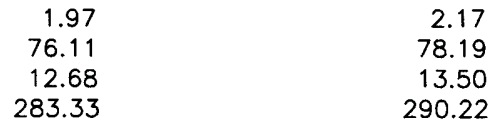
Fig. 5. Ion and electron measurements on 7 March 1997 between 21:10 and 21:50 UT. From top to bottom: energy-time spectrogram of electrons in the energy range $30 \mathrm{eV}$ to $10000 \mathrm{eV}$, energy-time spectrogram of protons in the energy range $30 \mathrm{eV}$ to $14000 \mathrm{eV}$ and proton pitch-angle. Fluxes are color coded. The satellite altitude $H$ in $\mathrm{R}_{\mathrm{E}}$, the invariant latitude $\Lambda_{0}$ in degrees, the magnetic local time MLT in hours, and the longitude: LONG in degrees, are given at the bottom of the figure. The electrons with energies lower than $70 \mathrm{eV}$ detected before 21:24 UT are secondary electrons
1. Equatorward of the cusp, from 21:10:00 to 21:26:00 UT, the satellite passed through a proton aurora showing an energy decrease from about 10000 to $4000 \mathrm{eV}$, when the latitude increased. This trend is very common in the dayside auroral oval, resulting from the adiabatic heating of the plasma of the inner plasma sheet followed by its strong grad $B$ drift in quasi-dipolar field topology (see Galperin et al., 1976, 1978; Sauvaud et al., 1981; Onsager and Mukai, 1995).

2. At energies lower than about $1 \mathrm{keV}$, high fluxes of protons were recorded. Particles at these energies drift

07/Mar/97 21:10:00

INTERBALL-AURORA ION
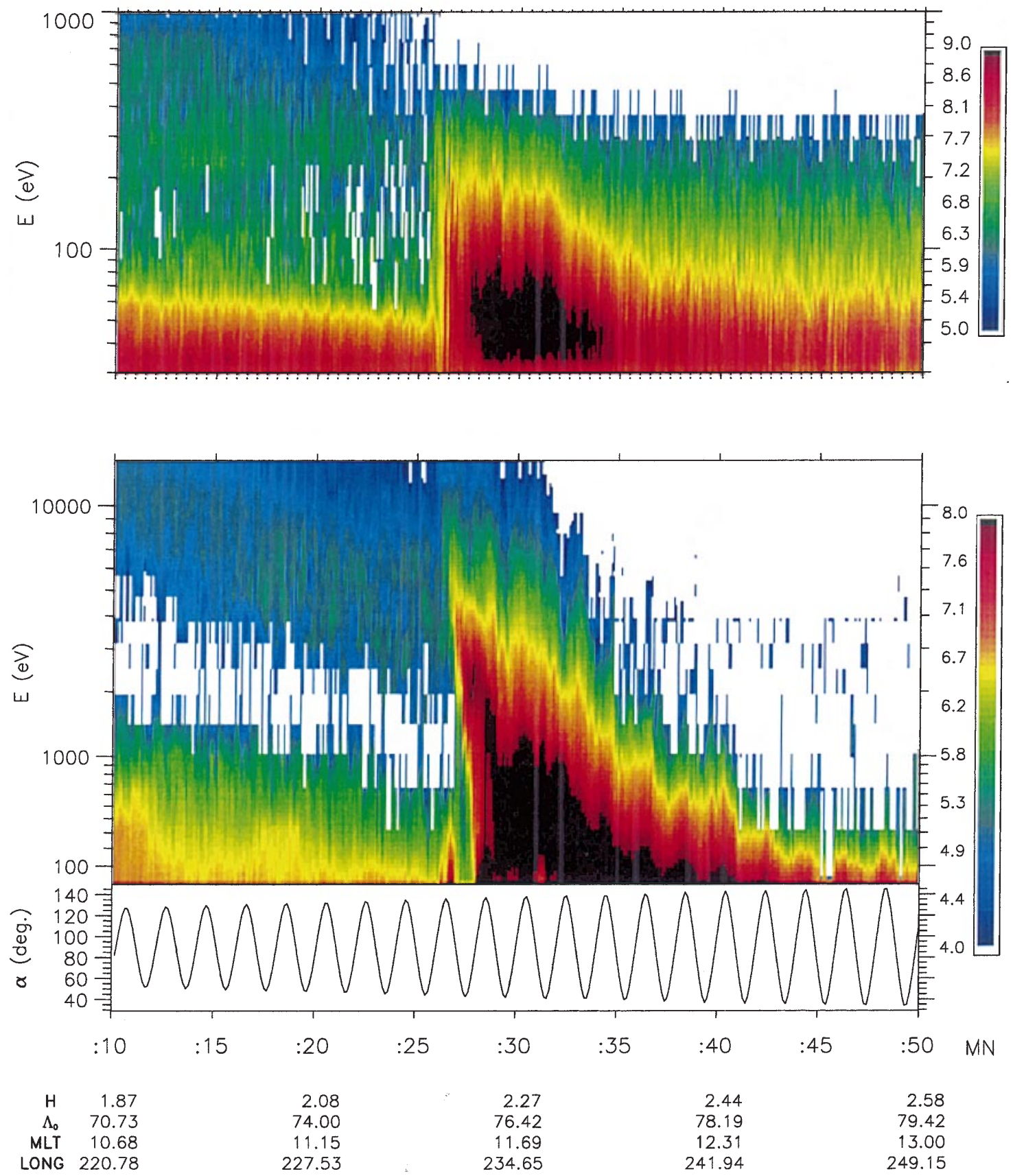

Fig. 6. Top panels: same as Fig. 5 for 24 February 1997 between 01:00 and 01:30 UT. The third panel from the top gives the oxygen fluxes and the bottom panel is the pitch-angle variation of both hydrogen and oxygen ions 
eastward under the effect of the electric field (convection and corotation). The origin of these low-energy ions can be either the ionosphere or the solar wind (see Sauvaud et al., 1981).

3. A gap in ion fluxes which displayed an energy dependence with latitude was detected equatorward of the cusp. No counts were measured, meaning the fluxes were below the measurement threshold, i.e., below $\approx 2 \cdot 10^{5} \mathrm{keV} /\left(\mathrm{cm}^{2} \cdot \mathrm{s} \cdot \mathrm{ster} \cdot \mathrm{keV}\right)$. This kind of gap is commonly observed in the morning and dayside auroral region onboard Interball-Aurora. To our knowledge, this feature has not been previously reported in the literature. A dedicated study is devoted to this structure, which results from an extremely long drift time $(>30 \mathrm{~h})$ for the ions which could be able to reach the gap; the ions are lost by wave-particle interactions and/or charge exchange (Kovrazhkin et al., 1998).

4. The electron energy-time spectrogram indicates the transition from auroral to magnetosheath-like electrons at 21:26:00 UT. Intense electron fluxes were detected after that time while ion fluxes of magnetosheath origin were detected 1 min later, first at energies of the order of $10 \mathrm{keV}$. The most intense electron precipitation lasts until about 21:32 UT.
5. At the equatorward edge of the cusp a clear energydependent dispersion of the protons was recorded. The low-energy cut-off can be assigned to the reconnection occurring at low latitude on the magnetopause (e.g., Hill and Reiff, 1977; Onsager et al., 1993; Alem, 1997). Coinciding with the energydispersed structure and with intense electron precipitation, upflowing low-energy protons were detected at 21:28:30 UT. We will discuss this specific structure later.

6. Between 21:26 and 21:45 UT the polar cusp hydrogen precipitation showed a classical energy-latitude dependence in the energy range $14 \mathrm{keV}$ to $\sim 100 \mathrm{eV}$. This energy-dispersed structure is already well documented for southward-directed interplanetary magnetic fields (e.g. Burch et al., 1982; Onsager et al., 1993). It results from the general northward convection of the plasma and from the large extension of the entry region on the magnetopause. A flux modulation with pitch angle was also detected. This modulation can be understood simply; starting from a fixed position on the magnetopause, only the ions with the same drift length in a direction perpendicular to the field line can reach the satellite position. For lowenergy ions the main drift is the $E \times B$ one, and the
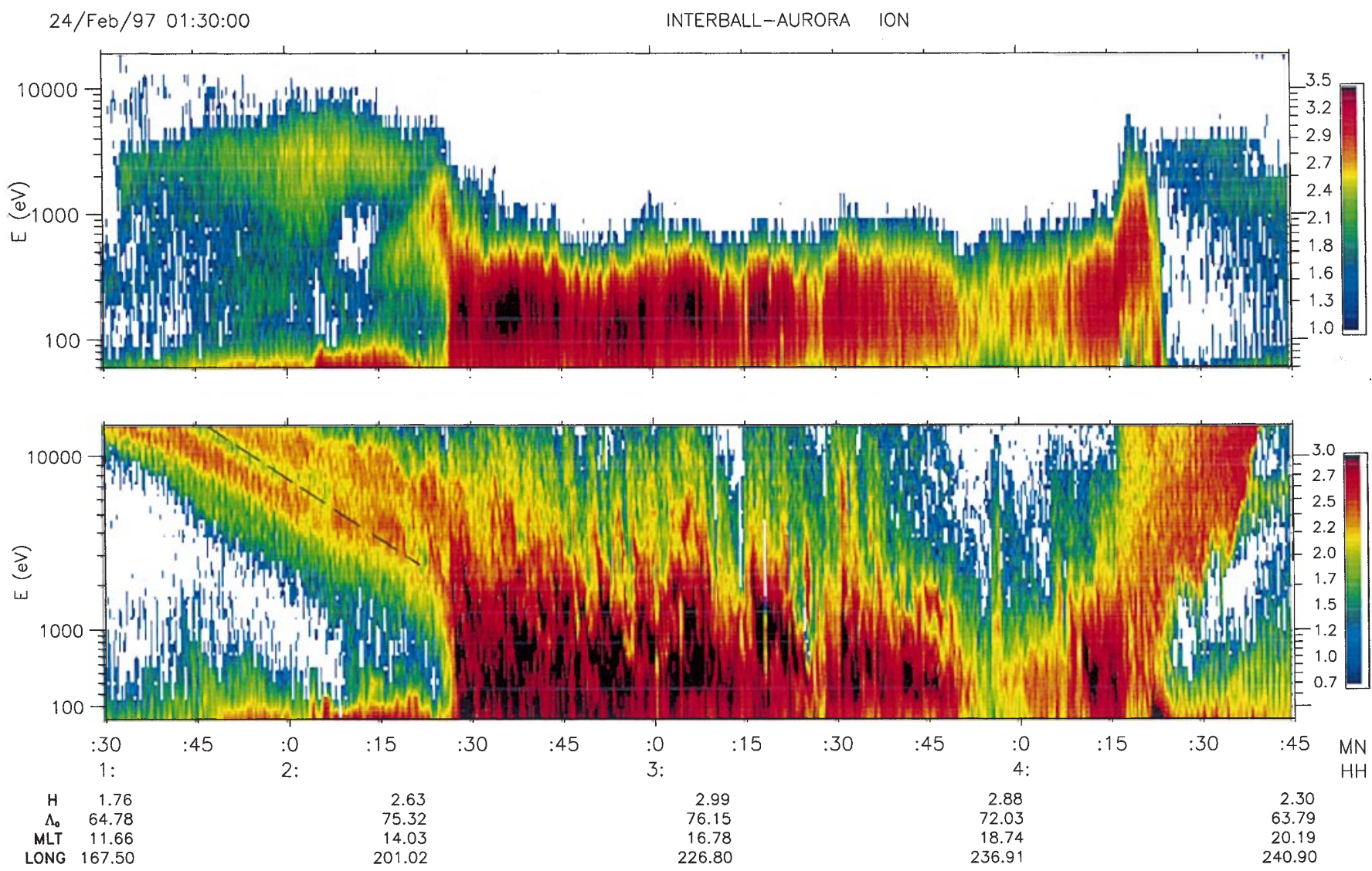

Fig. 7. Electron (top) and proton (bottom) energy-time spectrograms for a complete pass over the polar regions on 24 February 1997 between 01:30 and 04:45 UT. Energy fluxes are color coded. Note that between 02:15 and 04:30 UT the satellite was at altitudes greater than
$16000 \mathrm{~km}$. The dashed line at the right of the ion spectrometer gives the slope of the energy variation expected from the conservation of the second adiabatic invariant (Fermi-type acceleration) 
drift length is proportional to the time of flight between the magnetopause and the satellite. For a nearly fixed satellite position, the ION experiment is thus expected to measure low-energy particles at low pitch angles and higher-energy particles at higher pitch angles coming from the same magnetopause location (see Onsager et al., 1993; Alem, 1997). Note however that in the foregoing simplified description time-dependent effects are not taken into account.

Figure 6 presents another cusp crossing on 25 February 1997 (IMF $B_{z} \sim \mid \operatorname{sim}-2 \mathrm{nT}$ ), also in the noon sector (12-14.4 hours MLT) from low to high latitudes $\left(73^{\circ}-79.5^{\circ}\right)$. The main features described for Fig. 5 are easily recognizable. Oxygen ion fluxes are displayed here in the third panel from the top. Upwelling hydrogen ions were detected starting at 18:08 UT when the satellite encountered magnetosheath electrons and before magnetosheath protons were detected. Upwardflowing oxygen ions are detected simultaneously with the first hydrogen outflow. Inside the cusp proper, oxygen ion fluxes up to $2 \mathrm{keV}$ appeared each time the detector was downward-looking, starting at pitch-angles of about $140^{\circ}$. This indicates that the main heating mechanism was acting below the satellite, possibly accelerating the ions perpendicular to the magnetic field. Note that the occurrence of most energetic upflowing oxygen ions corresponds to the more intense electron precipitation. The detection of $\mathrm{O}^{+}$outflows in the cusp is in agreement with the statistical analysis of DE-1 satellite data performed by Kondo et al. (1990). These authors reported an enhancement of ion conics corresponding to the cusp and the cleft regions on the dayside. It must also be stressed that both cusp crossings showed high fluxes of protons, indicating an intense heating of ionospheric hydrogen. Perpendicular ion energization is caused by the essentially perpendicular components of electric fields oscillating within some frequency range (see the review by André and Yau, 1997). Several wave modes can cause perpendicular ion energization ranging from static structures and waves much below the gyrofrequencies to waves at and just above the ion gyrofrequencies to waves above the lower hybrid frequency (Chang and Coppi, 1981; Borovski, 1984; Klumpar, 1986; Lysak, 1986; André, 1997). A correlative study of ion heating detected by the ION
24/Feb/97 02:45

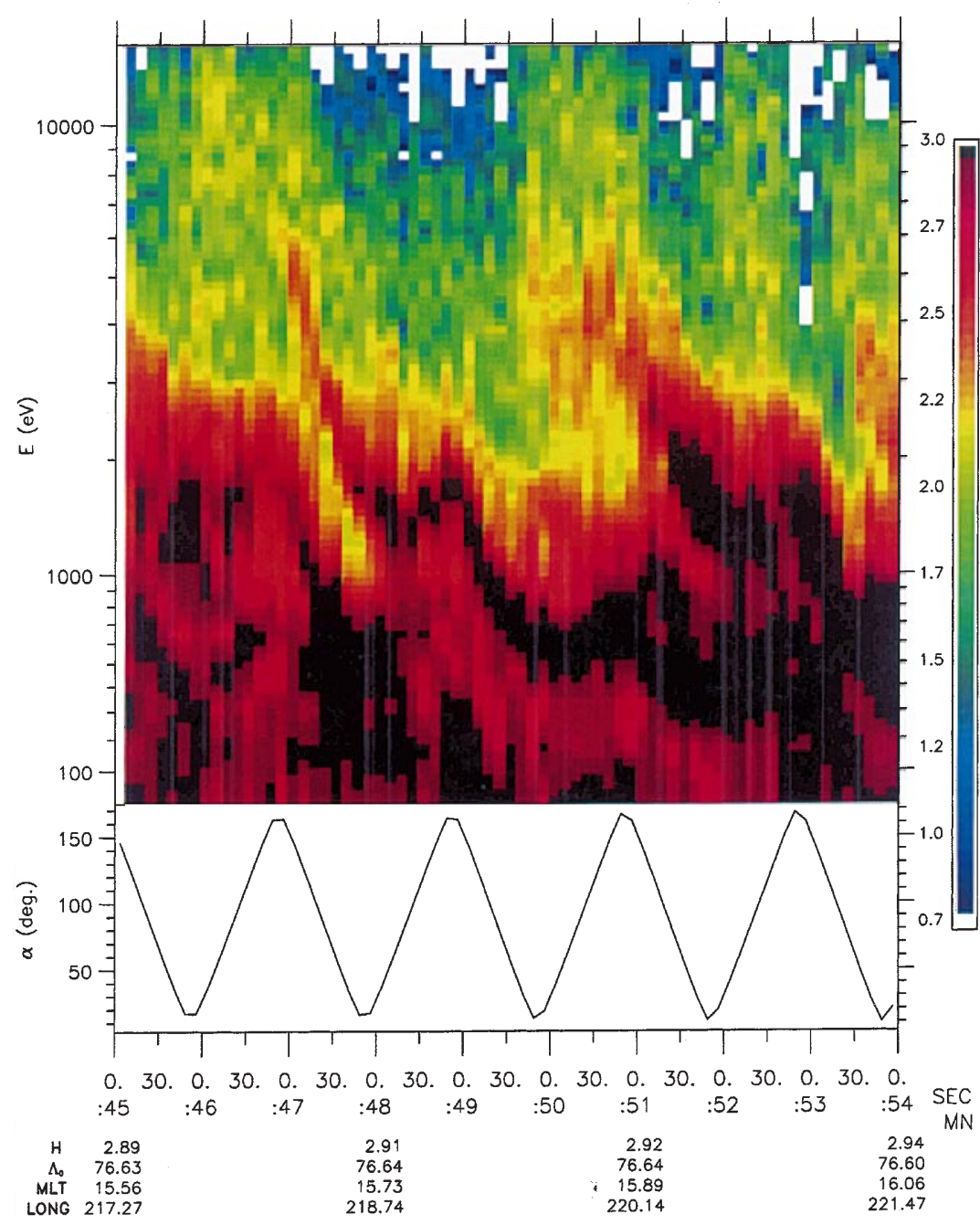

Fig. 8. Detail of Fig. 7. Energy-time spectrogram of protons between 02:45 and 02:54 UT on 24 February 1997. At the bottom, the pitch-angle of the ions. Note the serpentine structure starting around 02:47 UT and the two maxima seen between 02:50:30 and 02:53 UT 
experiment and low-frequency wave measurements performed onboard Interball-Aurora is underway (see, Perraut et al., 1998). It must be stressed that several mechanisms can concur to accelerate the ions, one of them being the electric field caused by the nonequipotentiality of reconnected field lines (Wing et al., 1997).

\subsection{Dusk polar cleft}

Ion and electron measurements performed on 24 February 1997 between 01:30 and 04:45 UT are given in Fig. $7\left(K_{\mathrm{p}}=0+, 2+\right)$. The satellite, coming from the dayside, first encountered an auroral proton population, then reached invariant latitudes higher than $74^{\circ}$ between approximately $02: 25$ and 03:45 UT in the dusk polar sector and passed again through the auroral precipitation region in the evening side of the oval. Between 01:00 and 02:30 UT and between 04:10 and 04:40, a very clear latitudinal energy variation for ions was detected, namely the $\mathrm{keV}$ ion average energy decreased when the latitude increased. This dispersion, seen at local times of about 11-13 MLT and then around 19 MLT, can be understood in terms of plasma heating inside the plasma sheet (see Galperin et al., 1978; Sauvaud et al., 1981). Electron measurements confirm that during these intervals the satellite was inside a weak plasma sheet electron population. The average ion energy can be expected to vary as $1 / \mathscr{L}^{2}$ due to conservation of the second adiabatic invariant:

$J=\oint V_{\|} d l \approx V \cdot \mathscr{L} \Rightarrow E \propto 1 / \mathscr{L}^{2}$,

where $\mathscr{L}$ is the field line length

A comparison of the data with the predicted $1 / \mathscr{L}$ dependence on the dayside expected from the T-89 magnetic field model (Tsyganenko, 1989) is indicated by a thick dashed line on the ion energy-time spectrogram in Fig. 7. The agreement is good, the model corresponds to $K p=2$. At 19:00 UT the agreement is much worse (not shown). We tentatively interpret this discrepancy as due to the dynamical behavior of the magnetosphere, since a substorm occurred at 04:00 UT after a very quiet period. Note that the electron average energy also strongly increased after 04:10 UT, although the latitudinal/local time extent of this high flux region was more limited than that of the ions. This difference can be linked to the local time of the satellite, 19 hours MLT, i.e., the local time sector where ions are expected to drift from the midnight sector while high-energy electrons are drifting eastward after substorm injection.

A very different region was detected at high latitudes. Here the ion and electron temperatures are much lower, and fluxes are high, comparable to those of the magnetosheath. We identify this region, encountered from $\sim 02: 23$ to $\sim 03: 47 \mathrm{UT}$, as the polar cleft. According to the Interball-Tail satellite located at dusk in the solar wind, the IMF $B_{z}$ component changed first from positive values $(5 \mathrm{nT})$ to values near zero and slightly negative at about 02:17 UT. Then, starting at $\sim 02: 45$ UT the IMF
$B_{z}$ became strongly negative $(\sim-5 \mathrm{nT})$ with a northward episode for 3 min just before 03:00 UT. In agreement with these measurements, a substorm growth phase was detected from provisional AE data starting several minutes after 03:00 UT. When Interball-Aurora entered the cleft, the IMF $B_{z}$ was already close to zero. The IMF $B_{x}$ component was positive $(\sim 6 \mathrm{nT})$ and the $B_{y}$ component negative ( -2 to $-3 \mathrm{nT})$.

Note that inside the cleft a succession of bursts of high energy $(10 \mathrm{keV})$ protons is detected (Fig. 7). As an example, Fig. 8 gives a detailed view of the proton energy-time spectrogram taken between 01:45 and 01:53 UT. Energy/pitch angle structures are now clearly apparent. In this time-interval, two main injections of ions were detected, both starting at high energies,

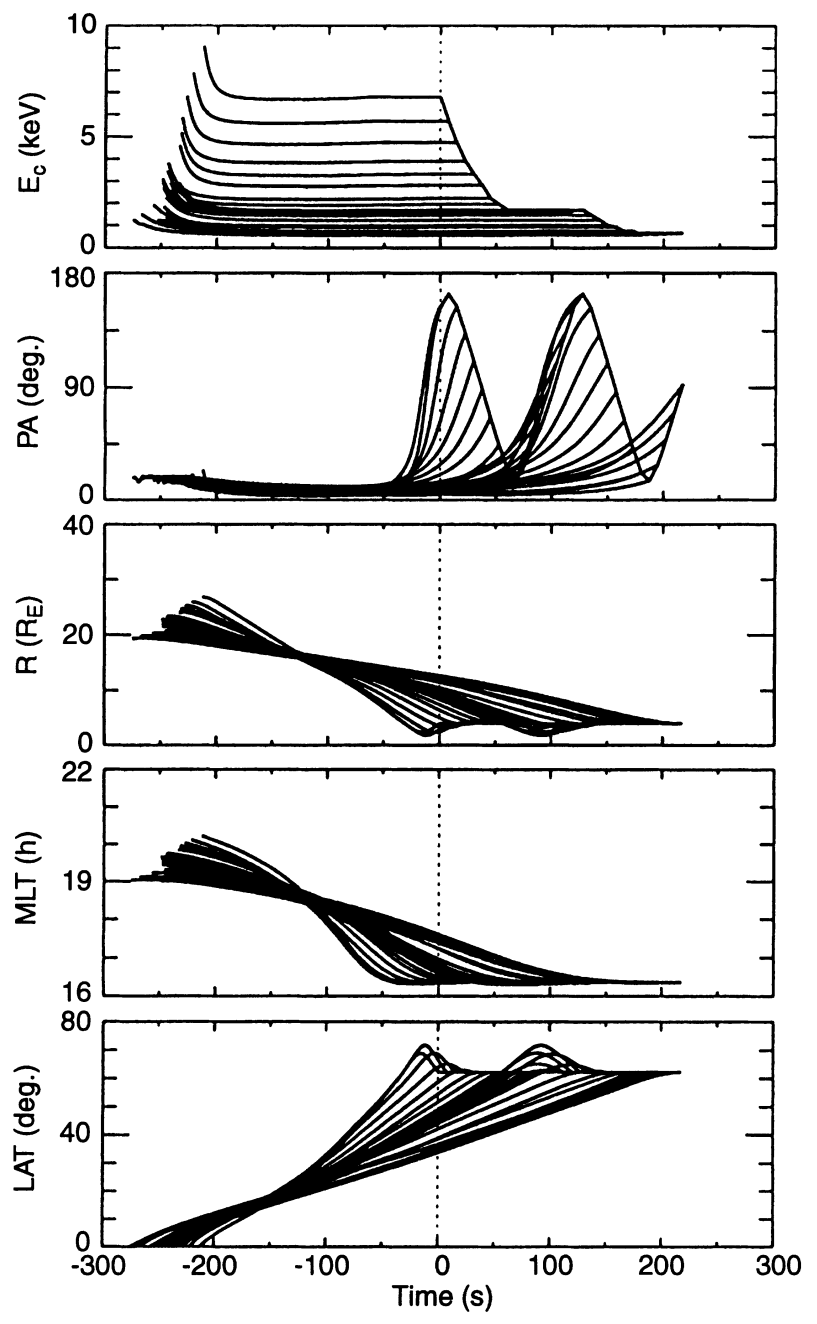

Fig. 9. Result of trajectories computed backward in time for the main ion energy/pitch angle structure displayed in Fig. 8. A 3D model of magnetic and electric fields is used (see text). At the top, the right black curve is the energy of the maximum ion fluxes between 02:47 $(T=0)$ and 02:50:30 UT. Energy variations when backward computations are performed are indicated. Next panel: ion pitchangle and its backward variations for selected energies. The three bottom panels give the variation of the radial distance, magnetic local time, and magnetic latitude of the particles forming the energy/pitch angle structure measured after $T=0$. Note the focal point at about $T=-130 \mathrm{~s}$ 
around $10 \mathrm{keV}$, showing a general energy decrease with time. During this 9-min time-interval, the satellite was nearly stationary near the apogee (magnetic local time 16 hours, invariant latitude $76.6^{\circ}$, altitude $2.95 \mathrm{R}_{\mathrm{E}}$ ). The ion structures showed a pitch-angle modulation: the energy, while generally decreasing, increased again when the spectrometer was viewing particles coming from the earth along the magnetic field, thus producing a kind of serpentine-shaped structure. Note that the apparent injection duration was about $5 \mathrm{~min}$, while the recurrence injection time was less, varying from 1.5 to $3.5 \mathrm{~min}$. This produces a characteristic double maximum at a given time in the energy-time spectrogram. The dispersion shape of the ion structure starting around 02:47 UT is very clear and reminiscent of a time of flight dispersion effect. We first tested this possibility by fitting a simple dispersion law $\left(t=t_{0}+L / v\right)$ through the experimental points disregarding the pitch-angle variations. The fit is good and indicates the ions were traveling a distance of the order of $25 \mathrm{R}_{\mathrm{E}}$ before reaching the satellite. We implicitly make the assumption that the particle pitch angle went close to zero quickly at higher altitudes. This is a reasonable assumption taking into account the variation of the earth's magnetic field (see Fig. 9). We have tested this with preliminary calculations using a full 3D model of particle trajectories in the T-89 (Tsyganenko, 1989) magnetic field model with a convection electric field model of Volland (1978) with a cross polar cap potential drop of $60 \mathrm{keV}$ [for a complete description, see Delcourt et al. (1989)]. The results are displayed in Fig. 9. The trajectories have been computed backward in time for about $250 \mathrm{~s}$. Initial energies and pitch-angles are taken into account. The main result is that all particles started simultaneously at $T_{0}-125 \mathrm{~s}$ from a region located $18 \mathrm{R}_{\mathrm{E}}$ from the earth, at a magnetic local time of 18.5 hours, i.e., very close to the dusk magnetopause. According to the model, the latitude of the injection is close to $20^{\circ}$. It is tempting to attribute this injection to sporadic and recurrent reconnection controlled by the IMF (e.g., Sauvaud et al., 1980; Newell and Meng, 1991). This result complements the work of Carlson and Torbert (1980), who found a source in the subsolar region from sounding rocket measurements using time-of-flight dispersion structures, and of Lundin et al. (1991) who similarly inferred sources in or near the dayside magnetopause boundary layer from VIKING data. Further studies on this subject are underway with Interball data.

\subsection{Dawn proton auroral precipitation}

The Interball-Aurora satellite's high apogee and relatively low inclination $\left(62.8^{\circ}\right)$ allow it to stay at nearly constant auroral latitudes for tens of minutes. We took advantage of this feature to study time-dependent phenomena over polar regions. This study was performed for an auroral pass of the satellite occurring on 27-28 September 1996, during a quiet period $(K p=2-, 2+)$. The satellite flew from the early morning side ( 2.4 hours
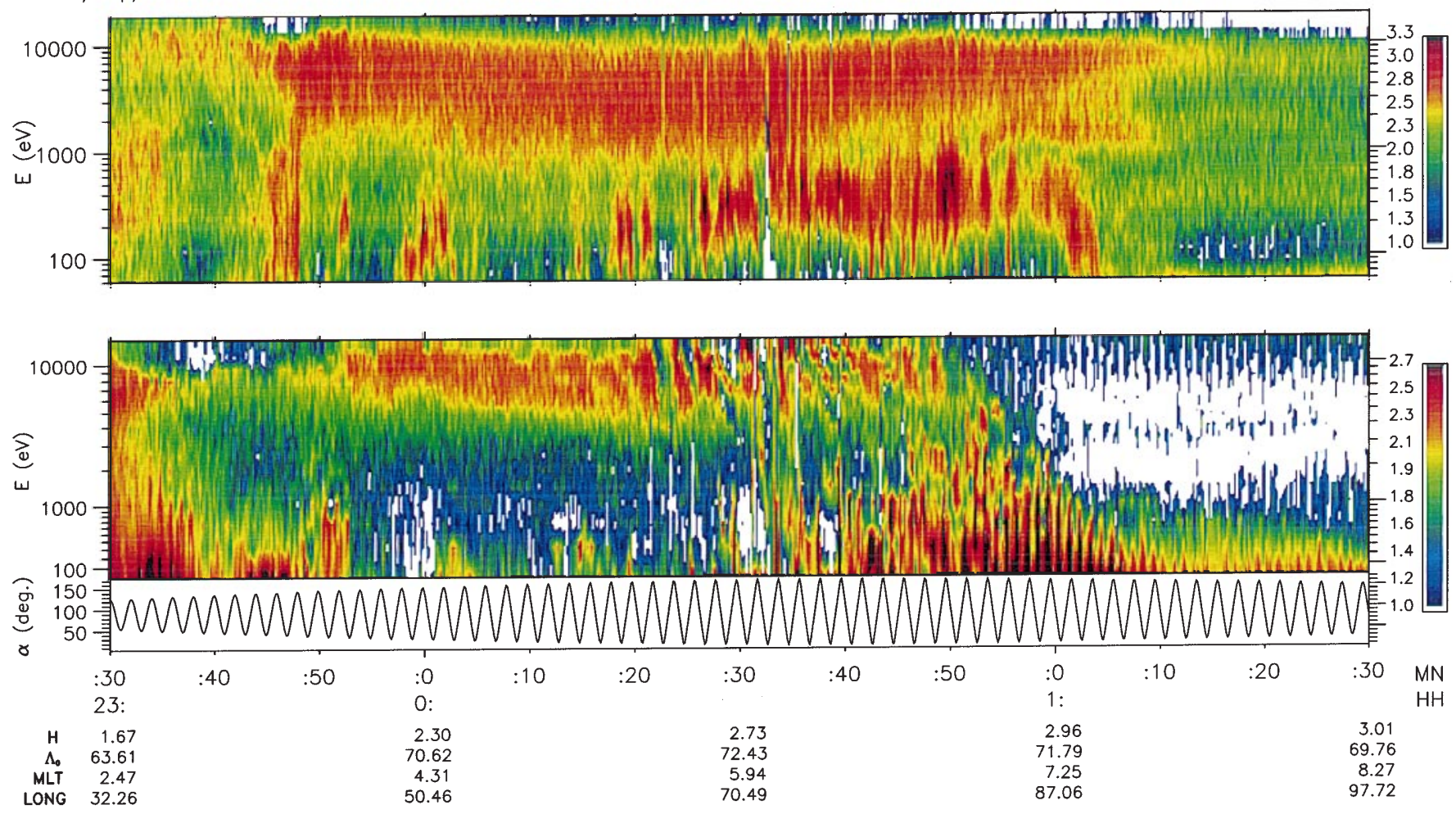

Fig. 10. Electron and proton energy-time spectrograms on 27-28 September 1996 between 23:30 and 01:30 UT. Energy fluxes are color coded. The bottom curve is the proton pitch angle 
MLT), near midnight, to the late-morning side of the oval (8.3 hours MLT), reaching the highest latitudes at $06 \operatorname{MLT}\left(72.50^{\circ}\right)$. A general view of the auroral particle data taken along the satellite path is presented in Fig. 10 which displays the electron and proton energy-time spectrograms, and the proton pitch angle variation.

The electron spectrogram shows several general features. (1) Fly between about 23:45 and 01:10 UT, a population of plasma sheet electrons with a temperature of several keV and intense energy fluxes; (2) during the same time-interval, the appearance of an electron population with energies lower than $1 \mathrm{keV}$; (3) on both sides of this traversal of the auroral region, equatorward of the main precipitation, the presence of a population of electrons extending to very low energies, $60 \mathrm{eV}$ here, around 23:45 and 01:03 UT, and (4) the continuation toward low latitudes of the plasma sheet population with reduced energy fluxes (between 23:30 and 23:45 UT, and between 01:10 and 01:30 UT).

The ion spectrogram exhibits several features which can be related to the electron one: (1) in the early morningside at low latitudes corresponding to the electron plasma sheet extension to low latitudes, an 8-keV proton nose as reported by Smith and Hoffman (1974) from observations onboard the Explorer 45 satellite near the equatorial plane, (2) bi-directional protons with energies lower than $3 \mathrm{keV}$ are simulta- neously detected, (3) a proton aurora with $\mathrm{keV}$ particle fluxes after 23:50 UT, first smoothly distributed around 7-10 keV, then starting to show clear energy dispersed structures at $\sim 00: 22$ UT, (4) outflowing low-energy protons, mainly between 00:22 and 00:42 UT, (5) an intense bi-directional low-energy ion population with a maximum energy reaching several $\mathrm{keV}$; this population was still detected with an energy not exceeding $500 \mathrm{eV}$ up to 01:30 UT, i.e., in the plasma sheet extension at small $L$ values. Starting around 00:58 UT there was a gap in proton fluxes between 1.5 and $7 \mathrm{keV}$; this kind of ion feature, very common in the morning auroral region, is linked to the excessive drift time of the particles which should reach the gap on the basis of their drift path geometry (Kovrazhkin et al., 1998).

Detailed electron and proton energy-time spectrograms between 1 and $14 \mathrm{keV}$, for the 10 -min period between 00:34 and 00:44 UT are given in Fig. 11 . Overlapping ion energy dispersion curves are clearly visible. They appear in the detector upper energy level about every $1.5 \mathrm{~min}$, at $\sim 00: 34: 33, \sim 00: 36: 00$, $\sim 00: 37: 42$, and finally $\sim 00: 39: 45$ UT. Each structure is detected for about $4 \mathrm{~min}$. Note that the last one stopped when a broader proton population began to be detected at $00: 41: 30$. The distribution function $f\left(v_{\|}, v_{\perp}\right)$ of the ions in the energy range 30 to $14000 \mathrm{eV}$ is given in Fig. 12 for the 1-min time-interval starting at 00:38:31
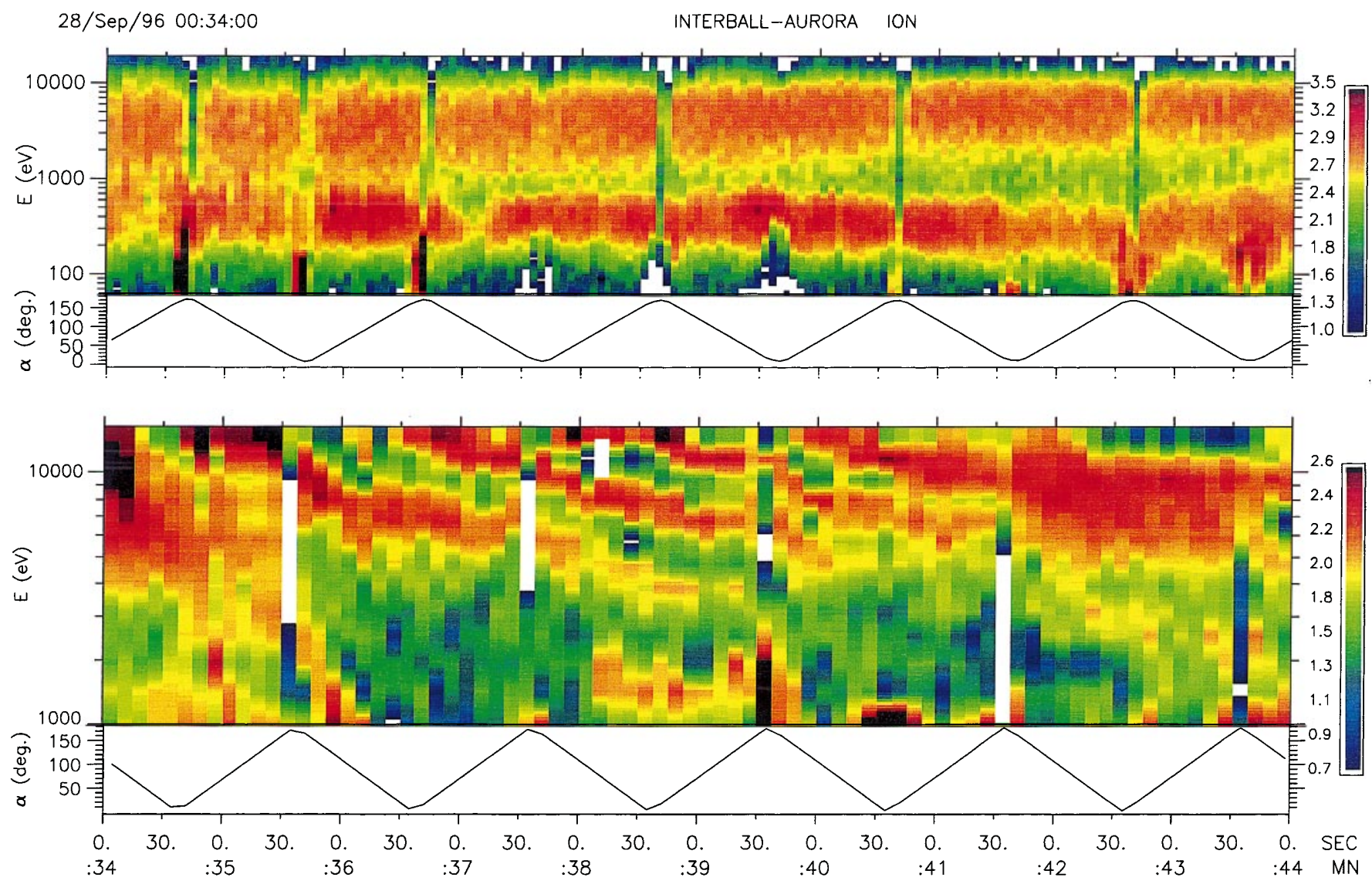

Fig. 11. Detail of Fig. 10 between 00:34 and 00:44 UT on 28 September 1996. Note the ion energy-time dispersions with the simultaneous detection of plasma sheet electrons $(E>1 \mathrm{keV})$ 
Start time: 28/ Sep/96 00:38:31 Vpar - Vperp (Ch1 ION2 H+)
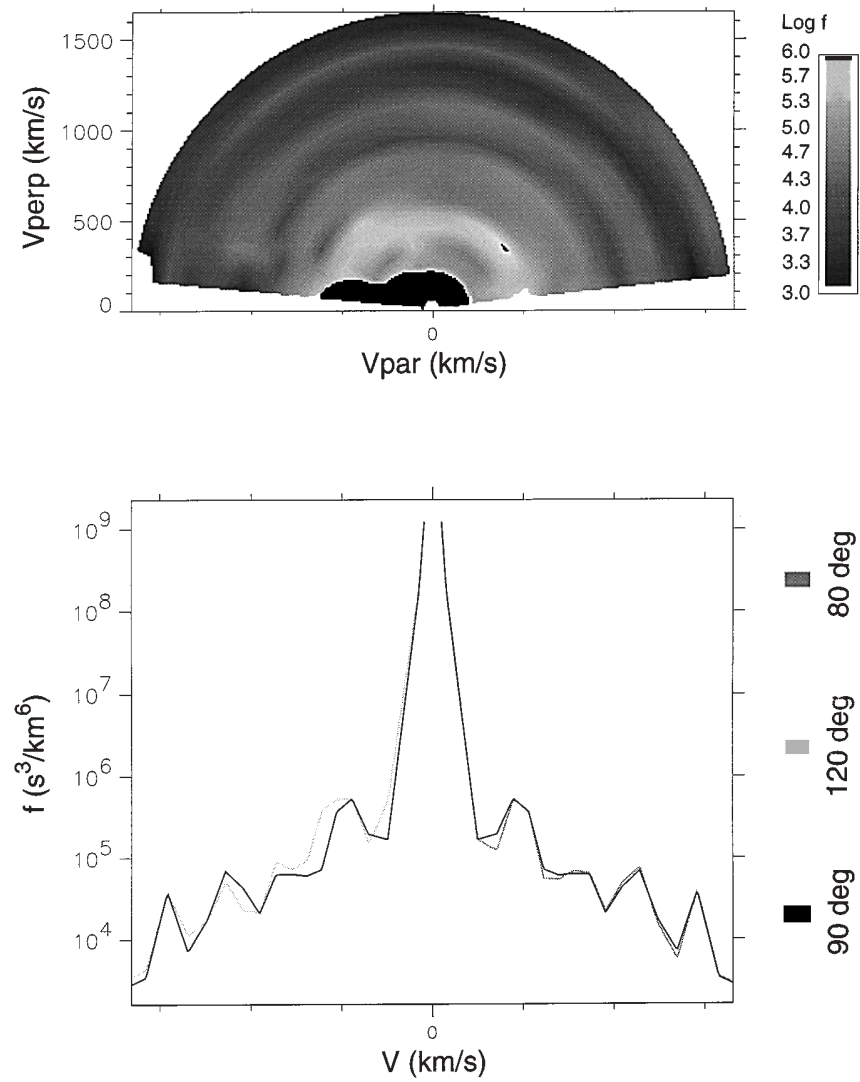

Fig. 12. Top: distribution function of the protons during a 1-min interval starting at 00:38:31 UT. Bottom: cuts of the $f\left(v_{\|}, v_{\perp}\right)$ for three pitch angles $\left(30^{\circ}, 90^{\circ}\right.$, and $\left.160^{\circ}\right)$

UT. Note that the energy-dispersed structure appears as a bump in the distribution function. Because of the slow satellite rotation, the maxima are slightly shifted from one distribution function cut to another. Note the low-energy ion outflow, clearly visible at $160^{\circ}$ pitchangles.

As in the case of the cleft energy-dispersed structures, we applied a simple time of flight model to the data. The fit gave good results and surprisingly long travel lengths, ranging from $77 R_{E}$ at 00:34 UT to $100 R_{E}$ at 00:37 UT. For the satellite location at 00:35 UT, the T-89 magnetic field model gives a field line length to the equatorial plane not exceeding $20 \mathrm{R}_{\mathrm{E}}$. However, the magnetic field model is an average, and furthermore the satellite is located in a very specific region at dawn, where a small change in latitude will cause the satellite to pass from quasi-dipolar field lines to field lines going to the magnetospheric tail through the flank. In order to confirm our preliminary time-of-flight calculations (which do not take into account the particle pitch angle), we used the following procedure: (1) Compute the trajectory of particles forming the energy dispersed structures backward in time in a 3D magnetosphere with electric and magnetic field models. (2) Iterate for increasing invariant latitudes searching for a possible initial time when the particles were all grouped in the outer magnetosphere. The final result is displayed in

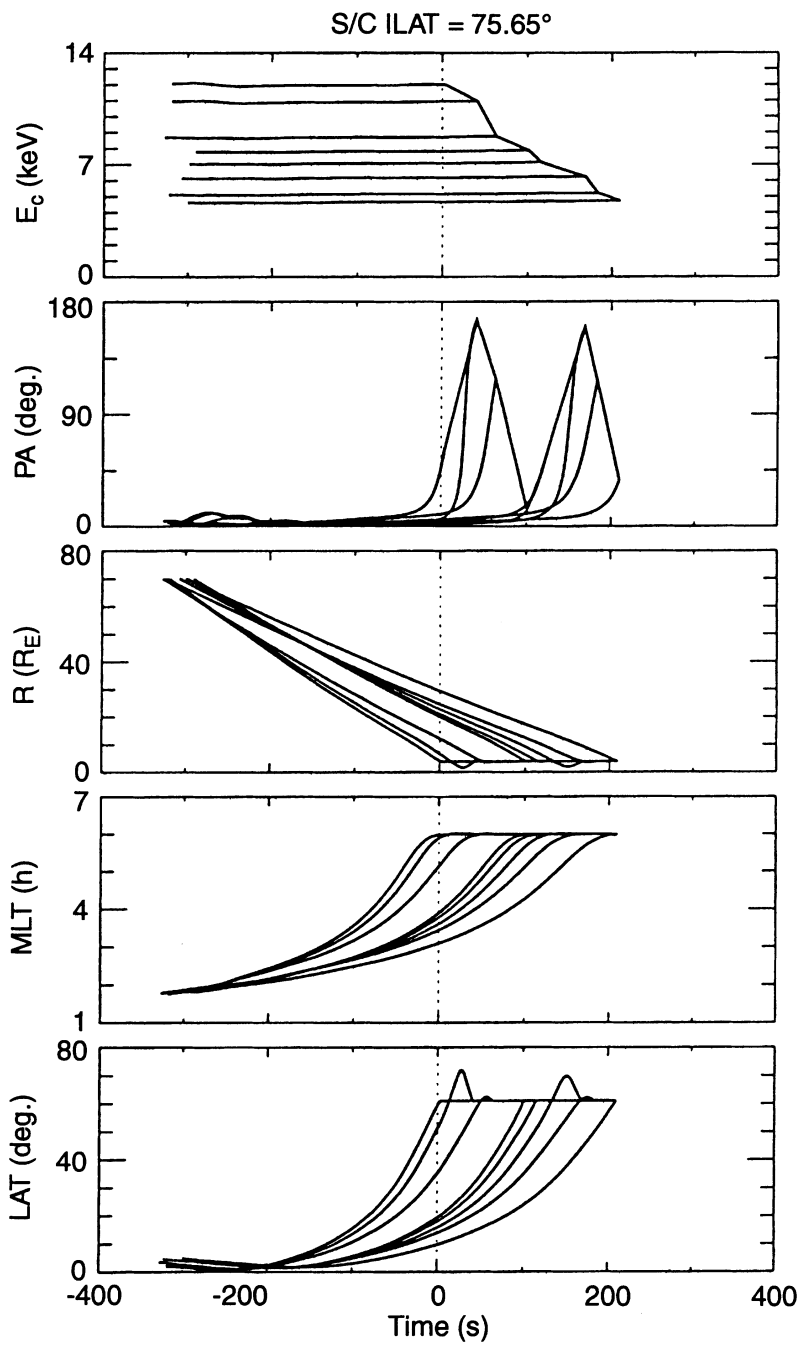

Fig. 13. Same presentation as Fig. 9 for the energy-pitch angle ion dispersion observed at 00:37 $(T=0)$ to 00:39:30 UT on 28 September 1996 (see Figs. 10 and 11)

Fig. 13 for the ion dispersion first appearing at 00:37 UT. Here, for an invariant latitude of $75.65^{\circ}$, the ions have a common origin in the tail, at radial distances of the order of $70-80 \mathrm{R}_{\mathrm{E}}$, a magnetic local time of 02 hours and a latitude of about $0^{\circ}$. This estimate is qualitatively well supported by the general aspect of the data. In Fig. 10 it must be noted indeed that energy-dispersed ion structures are first detected when the electron maximum energy reached a minimum, i.e., when the satellite was connected to the most distant plasma sheet it reached on that orbit. Other trials for lower latitudes of the measurement point gave different results with nonconverging curves, in disagreement with the time-offlight nature of the observed dispersion. Note that although the initial MLT is only 02 hours, it corresponds to the tail flank at $80 \mathrm{R}_{\mathrm{E}}\left(Y_{\mathrm{GSM}}=-35 \mathrm{R}_{\mathrm{E}}\right)$.

The ions are injected, then the precipitation stops and the cycle continues. For distances as large as $80 R_{E}$ we expect the ion orbits in the tail to be nonadiabatic. To obtain high fluxes inside the loss cone is difficult; only a very small part of the distribution function is pitch angle diffused (Delcourt et al., 1996). Another possibility is 
that a parallel electric field acts to align the particles. Such fields are expected at the interface of the plasma sheet and low-latitude boundary layer where the antisunward and sunward plasma flows mix. The flows shear and associated divergent electric fields can set up parallel electric fields and the boundary motion/instability could be linked to Alfvén or kinetic waves acting as a source modulation. More theoretical work is clearly needed fully to understand the observations.

\section{Summary}

The ION experiment has been returning auroral ion and electron data since the end of September 1996. The satellite drifts around the pole in about 9 months, so that a full local time coverage has in principle been available since July 1997. However, due to operational constraints, mainly satellite reorientations, another 9month period is needed to accomplish a good coverage. Periods of correlated measurements onboard InterballTail and Interball-Aurora both in the nightside or in the cusp/cleft have been identified and are under study. Already new results have been obtained with the ION experiment from measurements made in the altitude range 10 000-20 $000 \mathrm{~km}$. One is the detection of an ion gap in the morning and noon sectors due to an excessive drift time of the ions which could reach these regions. Also a clear determination of the dusk flank injection location of ions into the cleft has been deduced from data and from simple modeling. Finally, measurements made during quiet times inside a dawn proton aurora reveal that the source of the poleward boundary of the aurora is pulsating and located on the flank of the magnetosphere at distances of the order of $80 \mathrm{R}_{\mathrm{E}}$, presumably at the boundary between the antisunwardflowing LLBL and the plasma sheet. For the last two examples of results we have taken advantage of the near stationarity of the satellite near apogee. Other results can be found in the companion paper by Dubouloz et al. (1998), particularly concerning the ionospheric counterpart of velocity dispersed ion structures (VDIS) observed at the polar edge of the nightside auroral oval.

Acknowledgements. We are indebted to D. C. Delcourt for his participation in the modeling presented in this paper and to Y. I. Galperin and D. C. D. for fruitful discussions. The construction of the ION experiment was supported by the French space agency, CNES, under contract N0. CNES-208. We are grateful to J.-P. Thouvenin, D. Fournier, and G. Azoulay for their support during the preparation of the project. We thank the Russian Space Agency, Lavochkin Space Association, Babakin Center and the Space Research Institute of Moscow (IKI) for making the project a reality, under very hard conditions. The work of R. A. K. and K. G. A. was supported by grants from the Russian Foundation for Basic Research (RFBR), and by INTAS grants.

Topical Editor K.-H. Glassmeier thanks A. Johnstone and P. Daly for their help in evaluating this paper.

\section{References}

Alem, F., Etude du transport du plasma dans la region des cornets polaires: dynamique des ions et des distributions a basse altitude, Thèse de Docteur de l'Université Pierre et Marie Curie, Paris, 1997.

André, M., Waves and wave-particle interactions in the auroral region, J. Atmos. Terr. Phys., 59, 1687, 1997.

André, M., and A. Yau, Theories and observations of ion energization and outflow in the high-latitude magnetosphere, Space Sci. Rev., 80, 27, 1997.

Balsiger, H., P. Eberhardt, J. Geiss, A. Ghielmetti, H. P. Walker, D. T. Young, H. Loidl, and H. Rosenbauer, A satellite-borne ion mass spectrometer for the energy range 0 to $16 \mathrm{keV}$, Space Sci. Instrum., 2, 499, 1976.

Borovski, E., The production of ion conics by oblique double layers, J. Geophys. Res., 89, 2251, 1984.

Bosqued, J. M., H. Barthe, J. Coutelier, J. Crasnier, J. Cuvilo, J. L. Médale, H. Rème, J. A. Sauvaud, and R. A. Kovrazhkin, The lowenergy electron and ion spectrometers on the AUREOL-3 satellite: the SPECTRO experiment, Ann. Geophys., 38, 567, 1982.

Burch, J. L., P. H. Reiff, R. A. Heelis, J. D. Winningham, W. B. Hanson, C. Gurgiolo, J. D. Menietti, R. A. Hoffman, and J. N. Barfield, Plasma injection and transport in the mid-altitude polar cusp, Geophys. Res. Lett., 9, 921, 1982.

Carlson, C. W., and R. B. Torbert, Solar wind ion injections in the morning auroral oval, J. Geophys. Res., 85, 2903, 1980.

Chang, T., and B. Coppi, Lower hybrid acceleration and ion evolution in the supraauroral region, Geophys. Res. Lett., 8 , 1253, 1981.

Delcourt, D. C., C. R. Chappell, T. E. Moore, and J. H. Waite, A three-dimensional numerical model of ionospheric plasma in the magnetosphere, J. Geophys. Res., 94, 11 893, 1989.

Delcourt, D. C., J.-A. Sauvaud, R. F. Martin, and T. E. Moore, On the nonadiabatic precipitation of ions from the near-Earth plasma sheet, J. Geophys. Res., 101, A8, 17409, 1996.

Dubouloz, N, J.-J. Berthelier, M. Malingre, L. Girard, Y. Galperin et al., Thermal ion measurements onboard Interball auroral probe by the Hyperboloid experiment, Ann. Geophysicae, 16, 1070-1085, 1998

Galeev, A. A., Y. I. Galperin, and L. Zelenyi, The INTERBALL project to study solar-terrestrial physics, Cosmic Res., 34, 342, 1996.

Galperin, Y. I., N. V. Jorjio, R. A. Kovrazhkin, F. Cambou, J. A. Sauvaud, and J. Crasnier, On the origin of auroral protons at the dayside auroral oval, Ann. Geophys., 32, 117, 1976.

Galperin, Y. I., V. A. Gladyshev, N. V. Jorjio, R. A. Kovrazhkin, N. V. Sinitsin, F. Cambou, J. A. Sauvaud, and J. Crasnier, Adiabatic acceleration induced by convection in the plasmasheet, J. Geophys. Res., 83, 2567, 1978

Ghielmetti, A., Ein Ionenspektrometer zur messung der zusammensetzung und der energieverteilung des magnetosphärischen plasmas, PhD thesis, Bern University, 1974.

Hill, T. W., and P. H. Reiff, Evidence of magnetospheric cusp proton acceleration by magnetic merging at the dayside magnetopause, J. Geophys. Res., 82, 3623, 1977.

Klumpar, D. M., A digest and comprehensive bibliography on transverse auroral ion acceleration in the magnetosphere and ionosphere, AGU, Washington DC, 389, 1986.

Kondo, T., B. A. Whalen, A. W. Yau, and W. K. Peterson, Statistical analysis of upflowing ion beams and conic distributions at DE-1 altitudes, J. Geophys. Res., 95, 12091, 1990.

Kovrazhkin, R. A., J. A. Sauvaud, and D. Delcourt, Interball-2 observations of $0.1-10 \mathrm{keV}$ ion gaps in the diffuse auroral zone, Geophys. Res. Lett. in press, 1998.

Lundin R., I. Sandahl, J. Woch, and R. Elphingstone, The contribution of the boundary layer EMF to magnetospheric substorms, in Magnetospheric substorms, Eds. J. R. Kan, T. A. Potemra, S. Kokubun, and Iijima, AGU, Washington DC, 355, 1991.

Lysak, R. L., Ion acceleration by wave-particle interaction, ion acceleration in the magnetosphere and ionosphere, AGU, Washington DC, 261, 1986.

Newell, P. T., and C.-I. Meng, Ion acceleration at the equatorward edge of the cusp: low-altitude observation of patchy merging, Geophys. Res. Lett., 18, 1829, 1991. 
Onsager, T. G., and T. Mukai, Low-altitude signature of the plama sheet boundary layer: observation and model, Geophys. Res. Lett., 22, 855, 1995.

Onsager, T. G., C. A. Kletzing, J. B. Austin, and H. MacKiernn, Model of magnetosheath plasma in the magnetosphere: cusp and mantle particles at low altitudes, Geophys. Res. Lett., 20, 479, 1993.

Perraut, S., A. Roux, F. Darrouzet, C. de Vildary, M. Mogilevski, and $\mathbf{F}$. Lefeuvre, ULF wave measurements onboard the Interball auroral satellite, Ann. Geophys., this issue, 1997.

Sauvaud, J. A., Y. I. Galperin, V. A. Gladishev, A. K. Kuzmin, T. M. Muliarchik, and J. Crasnier, Spatial inhomogeneity of magnetosheath proton precipitation along the dayside cusp from the ARCAD experiment, J. Geophys. Res., 85, 5105, 1980.

Sauvaud, J. A., J. Crasnier, K. Mouala, R. A. Kovrazhkin, and N. V. Jorjio, Morning sector ion precipitation following substorm injections, J. Geophys. Res., 86, 3430, 1981.

Shelley, E. G., R. D. Sharp, R. G. Johnson, J. Geiss, P. Eberhardt, H. Balsiger, G. Haerendel, and H. Rosenbauer, Plasma composition experiment on ISEE-A, IEEE Trans. Geosci. Electron., 16, 266, 1978.
Shelley, E. G., D. A. Simpson, T. C. Sanders, H. Balsiger, and A. Ghielmetti, The energetic ion composition spectrometer (EICS) for the Dynamics Explorer-A, Space Sci. Instrum., 5, 443, 1981.

Shelley, E. G., A. Ghielmetti, E. Hertzburg, S. J. Battel, K. Atweggvon Burg, and $\mathbf{H}$. Balsiger, The AMPTE/CCE hot plasma composition experiment (HPCE), IEEE Trans. Geosci. Remote Sensing, 23, 241, 1985.

Smith, P. H., and R. A. Hoffman, Direct observations in the dusk hours of the characteristics of the stormtime ring current particles during the beginning of magnetic storms, J. Geophys. Res., 79, 966, 1974.

Tsyganenko, N. A., A magnetospheric magnetic field model with a warped current sheet, Planet. Space Sci., 37, 5, 1989.

Volland, H., A model of the magnetospheric convection electric field, J. Geophys. Res., 83, 2695, 1978.

Wing, S., P. T. Newell, and T. G. Onsager, Modeling the entry of magnetosheath electrons into the dayside ionosphere, J. Geophys. Res., 101, 13155, 1996. 